\title{
Dielectrophoretic Fabrication and Electron Microscopy Characterization of One-Dimensional-Nanomaterial-Based Devices
}

\author{
Lifeng Dong and Jun Jiao \\ Department of Physics, Portland State University, Portland, Oregon 97201, USA
}

One-dimensional (1D) nanomaterials have attracted great attention as building blocks for nanoscale electronics and optoelectronics. A number of 1D nanomaterials have been synthesized and studied [1]. However, it is still challenging to display 1D nanomaterials onto desirable locations for nanodevice fabrications. In this study, we report the development of an effective dielectrophoretic process to align 1D nanomaterials, including carbon nanotubes (CNTs) and NiSi nanowires, between two designated electrodes. Electron microscopy techniques in combination with electrical characterizations were used to explore the correlations between electrical properties of nanodevices and structural properties of the nanomaterials.

Prior to dielectrophoretic alignment, CNTs and NiSi nanowires were dispersed in deionized water containing $1 \mathrm{wt} \%$ sodium dodecyl sulfate (SDS) and ethanol solution, respectively. After periods of sonication and centrifugation, a drop of nanomaterial suspensions was applied onto a region consisting of several patterned electrodes. The electrodes were fabricated on silicon substrates coated with a $1-\mu \mathrm{m}$ thick thermal oxide layer. An alternating current electrical potential $(5-10 \mathrm{Vp}-\mathrm{p}, 5-10 \mathrm{MHz})$ was then applied onto two control electrodes for $1 \mathrm{~min}$. The substrates with aligned nanomaterials were soaked in deionized water for $30 \mathrm{~min}$ and dried with flowing nitrogen gas. The details of this process are described elsewhere [2-3].

Figs.1a-1c demonstrate that individual single-walled carbon nanotubes (SWCNTs), multi-walled carbon nanotubes (MWCNTs) and NiSi nanowires without other impurities can be dielectrophoretically aligned between two electrodes by optimizing the electrical field distribution and the concentration of nanomaterial suspensions. This proves that SDS molecules can functionalize the surface of CNTs and effectively separate bundles of nanotubes into individuals. With the increase of nanomaterial concentration, chained and branched nanomaterials can be formed as shown in Fig. 2. This implies that when an external electrical field is applied onto the NiSi nanowire suspension, nanowires are polarized with dipoles induced within the wire and aligned along the electrical field direction by the electrical torque. Then, due to the Coulomb force, if the neighboring nanowires are close enough to be electrically attracted, polarized nanowires attract the neighboring nanowires to form chains parallel to their long axes. The newly formed chain is then positioned by dielectrophoresis onto the top of an electrode as demonstrated in Fig. 2a. When a nanowire or a nanowire chain is positioned on top of the electrodes, a strong electrical field around the nanowire tips is induced by the nanowires' high aspect ratio and the small radius of the tips. The surrounding nanowires in the suspension tend to align along the radial directions of the nanowire to form nanowire branches as shown in Fig. 2b.

In addition to the electron microscopy characterization, electrical measurement on the fabricated testing structures was performed to investigate the dielectrophoretic alignment mechanisms and to understand the correlations between the fabrication conditions and the electric properties of the fabricated structures. Fig. 3a clearly shows that, besides metallic SW/MWCNTs and NiSi nanowires, individual semiconducting SWCNTs can be aligned between two electrodes. The on/off ratio is about $10^{5}$, which is comparable to the performance achieved by other back-gated nanotube transistors fabricated by other methods. Concerning the contact, our experiments suggest that it is more difficult to get good contact between the nanowires and the electrodes than between CNTs and electrodes. For instance, forty aligned NiSi nanowires were tested. None of them showed a detectable current signal above the noise background at picoampere levels. In an attempt to improve the physical contact between the nanowire and the electrode surfaces, we used an electron beam to irradiate both ends of the nanowires during SEM characterization. A typical I-V curve in Fig. 3b demonstrates that currents of several nanoamperes were obtained from the nanowires after the electron-beam irradiation. The 
nonlinear I-V behavior indicates that there are still potential barriers to carrier transport between the NiSi nanowire and the electrodes.

\section{References}

[1] Z. L. Wang, Nanowires and Nanobelts, Kluwer Academic Publisher, Norwell, MA, 2003.

[2] L. F. Dong, V. Chirayos, J. Bush, J. Jiao, et al., J. Phys. Chem. B 109 (2005) 13148.

[3] L. F. Dong, J. Bush, V. Chirayos, R. Solanki, J. Jiao, et al., Nano Lett. 5 (2005) 2112.

[4] The authors would like to thank J. Bush, V. Chirayos, S. Youkey, and J. Lo for helping with sample preparations. This research was supported by the Intel Corp. and the NSF under DMR-220926.
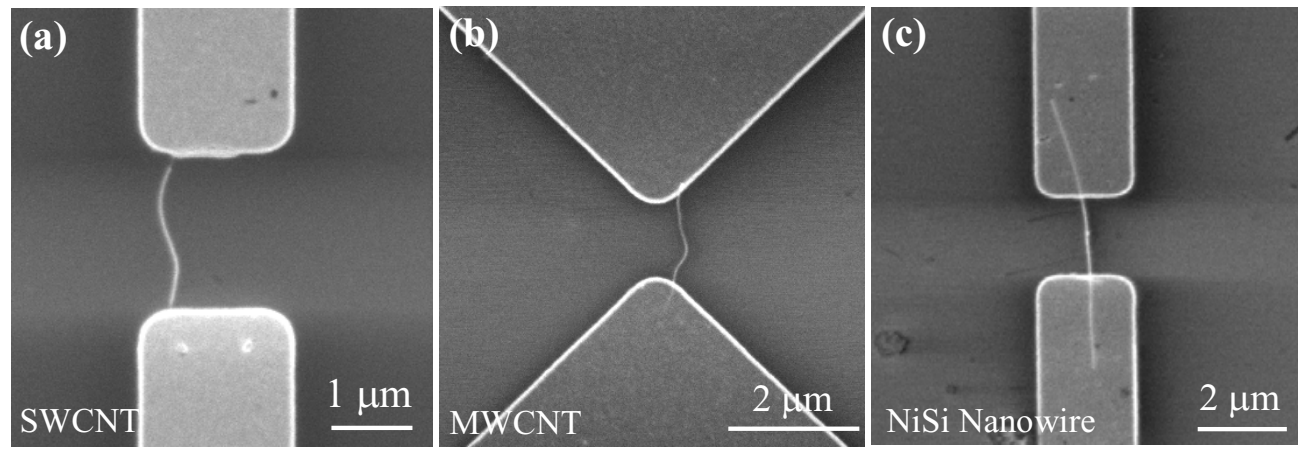

Fig. 1 SEM images of one individual SWCNT (1a), MWCNT (1b), and NiSi nanowire (1c) without other impurities aligned between two electrodes along electrical field directions.
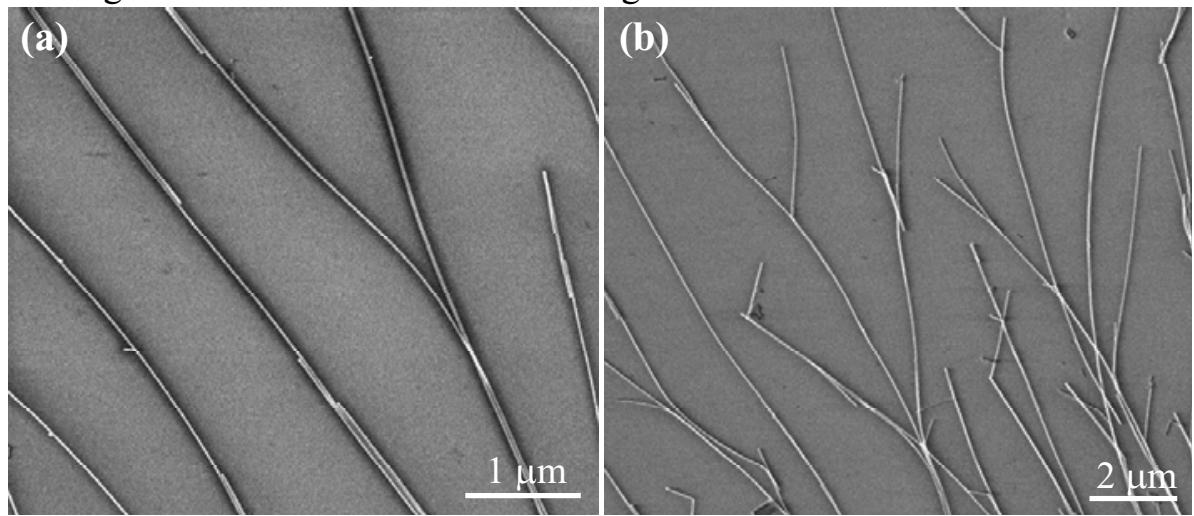

Fig. 2 SEM images of chained (2a) and branched (2b) NiSi nanowires. These phenomena were induced by the dipole-dipole interaction and the enhanced electrical field surrounding the aligned nanowires.
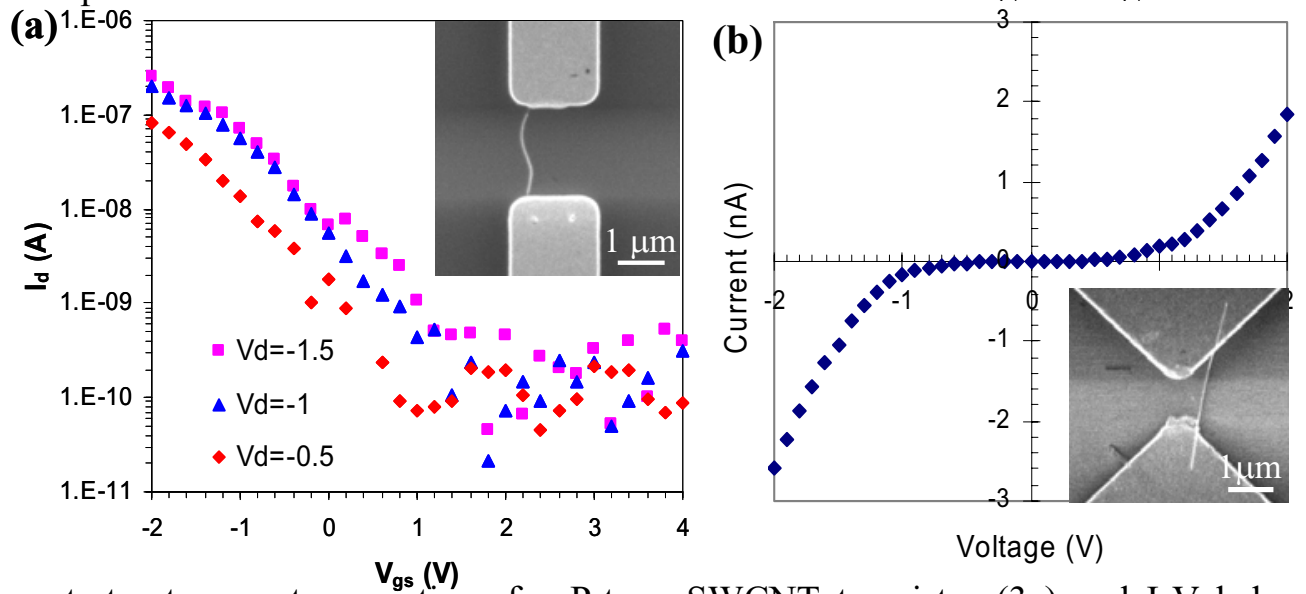

Fig. 3 demonstrates transport properties of a P-type SWCNT transistor (3a) and I-V behavior of a NiSi nanowire after e-beam irradiation (3b). Insets: SEM images of the measured nanostructures. 